\title{
Evaluation of the Ciba Corning Biotrack 512 coagulation monitor for the control of oral anticoagulation
}

\author{
I Jennings, R J Luddington, T Baglin
}

\begin{abstract}
The Ciba Corning Biotrack 512 coagulation monitor requires a minimal degree of technical expertise to operate, and is already in use for near-patient testing. This study evaluated the monitor for possible use in decentralised control of oral anticoagulant treatment. The monitor compared well with Manchester Reagent, suggesting that it could be used in areas where this thromboplastin is used for centralised control.

The inability of the monitor to allow for locally determined geometric mean normal prothrombin times in the calculation of the International Normalised Ratio (INR), and possibly the high International Sensitivity Index (ISI) of the thromboplastin used with the monitor, resulted in poor comparability with some other thromboplastins, particularly Thrombotest. These problems need to be addressed if the monitor is to be used for decentralised anticoagulant control.
\end{abstract}

Decentralised or "bedside" testing is being increasingly used to obtain rapid test results in several hospital departments, such as intensive care units and operating theatres. Such testing is also applicable outside hospitals in community health centres-for example, pregnancy testing and the monitoring of blood sugar in diabetes. ${ }^{1}$ With detailed guidelines recently published on the control and recommended doses of oral anticoagulants, ${ }^{2}$ coagulation testing and control of oral anticoagulant treatment could be carried out by general practitioners. This would relieve the increasing workload experienced by hospital laboratories. In this department the annual number of outpatient tests performed for oral anticoagulant control increased by over $40 \%$ since 1987 to almost 10000 tests a year. It could also lead to major financial savings in costs incurred from transporting samples or indeed patients from the community to hospital anticoagulant clinics. This approach requires a method for anticoagulant control which satisfies the criteria for decentralised testing-namely, the rapid production of results which can be accurately and reliably obtained by staff with a minimum of training and laboratory experience. $^{3}$
The Ciba Corning Biotrack 512 coagulation monitor is an automated technique for whole blood measurement of the prothrombin time, using capillary blood sampling. It is already used in operating theatres for preoperative monitoring of coagulation during liver transplantation, ${ }^{4}$ and a similar model, the Protrack Monitor 1000, correlates well with reference prothrombin time methods, both for normal subjects and patients receiving anticoagulation. ${ }^{5}$ This monitor has also been used for patient self-management of oral anticoagulant control in the United States. ${ }^{6}$

In this study the $\mathbf{5 1 2}$ monitor was used in a series of outpatient anticoagulant clinics in parallel with the existing method of anticoagulant control, the Nycomed capillary thrombotest reagent, to compare the international normalised ratios (INRs) obtained by each method. Subsequently, the performance of the $\mathbf{5 1 2}$ monitor was compared with several different commercial thromboplastins.

\section{Methods}

INRs were measured on 104 patients receiving warfarin for a variety of conditions, and 20 normal subjects. Capillary blood was obtained from a fingerprick sample. Venous blood was collected into a $1 / 10$ th volume of $3.8 \%$ trisodium citrate, centrifuged at $2500 \times g$ for five minutes, and separated into plastic tubes before the plasma INRs were measured.

Measurement of the prothrombin time by the $\mathbf{5 1 2}$ monitor was as follows: a prothrombin time cartridge, consisting of a capillary channel leading into a chamber containing dried thromboplastin (Ciba Corning, Halstead, Essex; batch P80236) was inserted into the monitor to warm to $37^{\circ} \mathrm{C}$. A drop of capillary blood was added to the sample application well. The blood was drawn by capillary action into the monitor where it rehydrated the thromboplastin reagent. As clot formation occurred, capillary blood flow ceased, and this was detected by variation in light scatter of the red blood cells. The monitor then displayed both the prothrombin time and an INR calculated from stored values for the mean normal prothrombin time and the ISI of the thromboplastin reagent used.

Capillary thrombotest was carried out on the same fingerprick sample. Blood $(50 \mu \mathrm{l})$ was aspirated and added to $250 \mu \mathrm{l}$ of capillary thrombotest reagent (Nycomed, Oslo Norway; batch 003002 ) at $37^{\circ} \mathrm{C}$. The clotting end point 
was measured manually by tilt-tube technique. The INR was calculated from the manufacturer's chart.

Plasma INRs were determined with different . thromboplastins on a $\mathrm{KC10}$ coagulometer (Baxter Dade, Dudingen, Switzerland) as the determination of INR from clotting times obtained with this coagulometer has been shown to compare closely with manual methods. ${ }^{7}$ The following techniques were used: For Thromboplastin-IS (Baxter, Dade; batch 515.014), Simplastin (Organon Teknika, Boxtel, Holland; batch 10126750), and PT-Fib reagent (Instrumentation laboratories, Ascoli Piceno, Italy; batch I0500149), 0.1 ml plasma was added to a sample cuvette. Pre-warmed thromboplastin $(0.2 \mathrm{ml})$ was added to the sample and the prothrombin time determined. For Manchester reagent (Manchester Thrombosis Research Foundation, Stockport), $0 \cdot 1 \mathrm{ml}$ plasma was added to the sample cuvette, followed by $0.1 \mathrm{ml}$ thromboplastin. Finally, $0.1 \mathrm{ml} 0.025 \mathrm{M}$ calcium chloride was added, and the clotting time measured.

For venous thrombotest $0.03 \mathrm{ml}$ plasma was added to $0.25 \mathrm{ml}$ venous thrombotest reagent (Nycomed; batch 003002, reconstituted in 3.2 $\mathrm{mM}$ calcium chloride) and the clotting time was measured.

The INRs displayed by the 512 monitor were automatically calculated, based on a stored mean normal prothrombin time of 12 seconds and an ISI recorded on the test cartridge.

Capillary and venous thrombotest INRs were determined from the manufacturer's chart. For all thromboplastins, including the 512 monitor and thrombotest reagent, the INR was also calculated by the UK NEQAS recommended method (Steering Committee letter to participants, June 1989) from the equation:

$$
\text { INR }=\left(\frac{\text { PT patient }}{\text { geometric mean normal PT }}\right) \text { ISI }
$$

where the mean normal PT was calculated as the geometric mean of the prothrombin times of 20 normal subjects (GMNPT), and the ISI was provided by the manufacturer.

A "consensus" INR was calculated as the mean INR for each sample derived from all methods.

Precision was assessed by determining the coefficients of variation (CV) for low and high INR control preparations obtained from the manufacturer. The CVs were calculated from the standard deviation/mean of 20 samples of each control.

The correlation coefficient is a measure of relation and not agreement between assays measuring the same parameter; calculation of the mean difference between assay measurements and the standard deviation of this mean is a more appropriate measure of agreement. Therefore, statistical evaluation was carried out by analysis of method of comparison, as described by Bland and Altman. ${ }^{8}$ The mean difference between individual patient INRs measured by different
Table 1 Comparison of INR results from 512 monitor and capillary thrombotest

\begin{tabular}{lll}
\hline & 512 INR & Capillary thrombotest \\
\hline Mean INR & 2.44 & $3.05^{\star}$ \\
Median INR & 2.28 & 2.80 \\
Minimum & 0.89 & 1.20 \\
Maximum & 5.97 & 8.00 \\
Range & 5.08 & 6.80 \\
\hline
\end{tabular}

${ }^{\star} \mathrm{p}<0.001$ (student's $t$ test).

thromboplastins was calculated, and the standard deviation of this mean was used to obtain limits of agreement-that is the potential variation in an individual measurement, when using different thromboplastins. Thromboplastins giving a positive mean difference show a relative tendency to overestimate the degree of anticoagulation. Conversely, a negative mean difference indicates a relative tendency to underestimate the INR.

\section{Results}

PRECISION

Coefficients of variation measured for the control preparations on the 512 monitor were mean INR $0 \cdot 79, \mathrm{CV} 7 \cdot 5 \%$, and mean INR $4 \cdot 3, \mathrm{CV}$ $4.5 \%$.

\section{COMPARABILITY}

INRs on 104 anticoagulated patients were measured using the 512 monitor and capillary thrombotest reagent. The calculated INR from the 512 monitor and the INR determined from the manufacturer's chart for thrombotest reagent were compared. Results are shown in table 1 and the mean difference and limits of agreement are shown in figure 1 . These show a significant underestimation of the INR by the 512 monitor relative to capillary thrombotest, and a potential difference in an individual INR measurement of up to $2 \cdot 17$ INR units.

The GMNPTs for the 512 monitor, capillary and venous thrombotest, and for four other thromboplastins are shown in table 2, together with the manufacturers' ISI for each method.

The mean difference from the consensus INR and the limits of agreement for each thromboplastin are shown in table 3 . The greatest difference from the consensus INR was

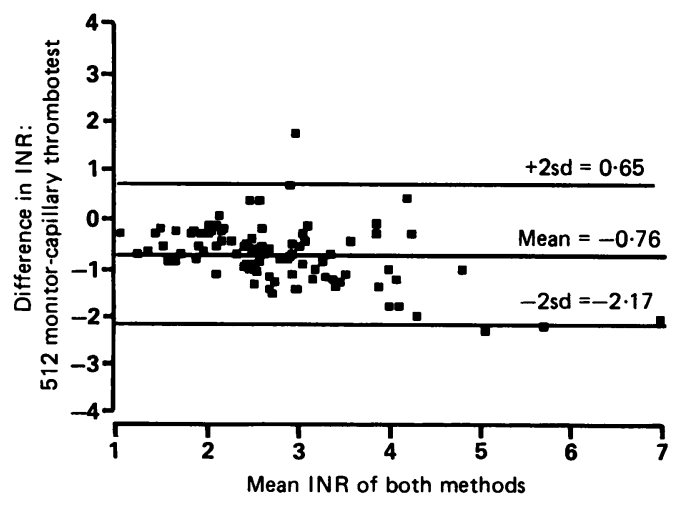

Figure 1 Comparison of 512 monitor and capillary thrombotest. The tendency of the 512 monitor to underestimate the relative INR is shown by the mean difference of -0.76 . 
Table 2 ISI and mean normal prothrombin time for all thromboplastins

\begin{tabular}{lll}
\hline $\begin{array}{l}\text { Thromboplastin } \\
\text { reagent }\end{array}$ & ISI & $\begin{array}{l}\text { Geometric } \\
\text { mean }(n=20)\end{array}$ \\
\hline 512: & & \\
Machine-calculated INR & 2.07 & $12.00^{\star}$ \\
GMNPT-calculated INR & 2.07 & 11.45 \\
Capillary thrombotest & 0.96 & $33.50 \dagger$ \\
Capillary thrombotest: & & \\
GMNPT-calculated INR & 0.96 & 34.90 \\
Venous thrombotest & 0.98 & $34.50 \dagger$ \\
Venous thrombotest: & & \\
GMNPT-calculated INR & 0.98 & 35.16 \\
Thromboplastin-IS & 1.24 & 14.00 \\
PT-Fib Reagent & $1.53 \ddagger$ & 15.48 \\
Simplastin & 1.23 & 13.38 \\
Manchester Reagent & 1.09 & 13.98 \\
\hline
\end{tabular}

*Pre-determined by manufacturer.

†From manufacturer's chart.

IISIs determined for manual and Automated Coagulation

Laboratory (ACL) methods; manual ISI used.

achieved with the 512 monitor INR at -0.35 INR units, and with capillary thrombotest at 0.44 INR units. The GMNPT INR calculated by monitor was comparable with the consensus INR (mean difference -0.09). The widest limits of agreement with the consensus INR were observed with the monitor INR, regardless of whether this was calculated by machine or manually.

The Manchester Reagent seemed the most comparable with the INR calculated by machine (512) and therefore a direct comparison was made (fig 2 ). The close comparability was confirmed with a mean difference in INRs of -0.07 .

\section{Discussion}

The first stage in evaluation of any new technique for decentralised testing is full evaluation of that technique by trained laboratory staff, and comparison with existing, quality controlled methods. The 512 monitor was easy to use, requiring no expertise and minimal training. Variables such as temperature control, subjective end point analysis, and accurate measurement of blood and reagent volumes have been removed, so that with adequate quality control, the instrument should prove suitable for decentralised testing.

The initial comparison between INRs obtained from the 512 monitor and capillary thrombotest showed poor comparability, with the 512 underestimating the INR by a mean of 0.76 INR units relative to the thrombotest reagent. The limits of agreement indicated that

Table 3 Comparison of consensus mean INR and INRs derived from individual thromboplastins

\begin{tabular}{llll}
\hline & $\begin{array}{l}\text { Mean }(S D) \\
\text { difference } \\
\text { (reagent- } \\
\text { consensus) }\end{array}$ & \multicolumn{2}{l}{$\begin{array}{l}\text { Limits of agreement } \\
\text { (mean }+/-2 S D)\end{array}$} \\
\cline { 3 - 4 } Reagent & $-0.35(0.50)$ & Upper & Lower \\
\hline 512 INR & $-0.09(0.50)$ & 0.65 & -1.35 \\
GMNPT INR & $0.44(0.36)$ & 0.91 & -1.09 \\
Capillary thrombotest & $0.31(0.33)$ & 1.16 & -0.28 \\
GMNPT INR & $0.02(0.34)$ & 0.97 & -0.35 \\
Venous thrombotest & $-0.03(0.33)$ & 0.70 & -0.66 \\
GMNPT INR & $-0.06(0.32)$ & 0.63 & -0.69 \\
Thromboplastin-IS & $0.22(0.36)$ & 0.58 & -0.70 \\
PT-Fib reagent & $-0.22(0.36)$ & 0.94 & -0.50 \\
Simplastin & $-0.25(0.35)$ & 0.50 & -0.94 \\
Manchester Reagent & & 0.45 & -0.95 \\
\hline
\end{tabular}

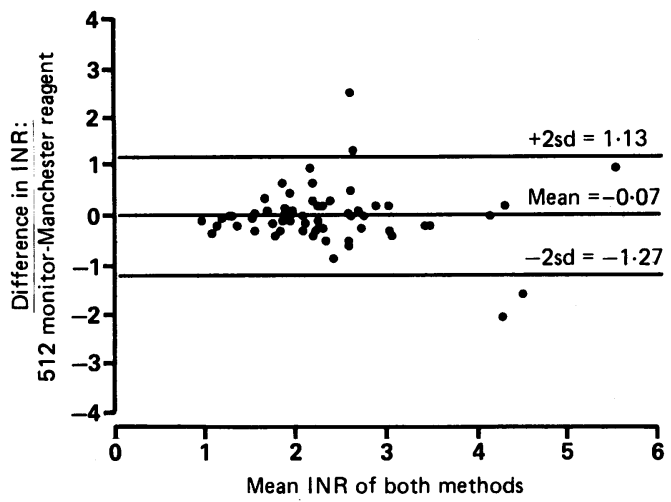

Figure 2 Comparison of 512 monitor and Manchester Reagent, showing close comparability of methods.

the INR obtained from the 512 and the capillary thrombotest reagent may differ by up to $2 \cdot 1$ INR units (mean difference $+2 S D$ ). As both different methods and different thromboplastins were used in deriving these INRs the performance of the 512 coagulometer and four thromboplastins, together with venous and capillary thrombotest, were evaluated. For each reagent (including the 512) the mean difference from consensus INR was calculated. Four of the 10 methods (including thrombotest) tended to overestimate the INR compared with the consensus, while six (including the 512) tended to underestimate the INR compared with the consensus. The thromboplastins most comparable with the 512 were Manchester Reagent and Simplastin. When the monitor INRs were compared with Manchester Reagent alone there was close comparibility (mean difference -0.07 ). Thus in this study the 512 INR is most comparable with the Manchester Reagent INR and least comparable with the thrombotest INR. When the 512 INR was derived from the GMNPT it was comparable with the consensus INR (mean difference -0.09 ). There is no facility within the monitor, however, to change the calculation of the INR, necessitating manual calculation which complicates what would otherwise be a simple to read measurement of anticoagulation.

The limits of agreement between the consensus INR and the different thromboplastins ranged from -1.35 to $1 \cdot 16$, indicating that an INR can differ from the consensus by more than the full therapeutic range of anticoagulation ( 2.0 to 3.0 by $\mathrm{BSH}$ criteria ${ }^{2}$ ) depending on the thromboplastin. The greatest limit was $1 \cdot 35$ INR units from the concensus INR in the case of the 512 INR. When the 512 INR was calculated from the GMNPT the mean difference from the consensus INR was minimal and the greatest limit from the consensus mean was reduced to $1 \cdot 09$. This wide limit of agreement might be expected as the ISI for the thromboplastin reagent was calculated as 2.07 , which as a particularly high ISI may result in poor coefficients of variation and precision of results. ${ }^{9}$ The precision of the instrument, however, was shown to be good for both low and high INR values, with coefficients of variation of $7.5 \%$ or less. The wide limit of agreement is therefore not readily explicable 
but may result from the monitor underestimating high INR values (INR $>4 \cdot 0$ ). This seemed to be a tendency with the machine, but the number of patients falling within this range was small and this could not be shown statistically.

In conclusion, the 512 monitor may be a suitable instrument for decentralised anticoagulant control as it is easy to use and precise. It would be particularly applicable for use in areas where centralised control is carried out using a similarly performing thromboplastin, such as Manchester Reagent. In its present form it would not be applicable in areas with centralised control using thromboplastins such as thrombotest. The latter incompatibility might be reduced if the facility for changing the GMNPT were introduced, but close comparibility with thromboplastins such as thrombotest would probably require a change of the thromboplastin incorporated in the $\mathbf{5 1 2}$ coagulometer. These factors need to be considered if the monitor is to be critically evaluated for decentralised control of oral anticoagulation.

1 Hailey DM, Lea AR. Developments in near-patient testing. Med Lab Sci 1990;47:319-25.

2 British Society for Haematology, British Committee for Standards in Haematology, Haemostasis and Thrombosis Task Force. Guidelines on oral anticoagulation: second edition. J Clin Pathol 1990;43:177-83.

3 Rachel JM, Plapp FV. Bedside blood grouping. Med Lab Sci 1990;47:330-6.

4 Luddington RJ, Smith M. Bedside PT and APTT monitoring during liver transplantation using the Biotract 512 coagulation monitor-preliminary findings. Eur J Anaesth 1990;2:31.

5 Lucas FV, Duncan A, Jay R, et al. A novel whole blood capillary technic for measuring the prothrombin time. $\mathrm{Am}$ capillary technic for measuring

6 Ansell J, Holden A, Knapie N. Patient self-management of oral anticoagulation guided by capillary (fingerstick) whole blood prothrombin times. Arch Intern Med 1989; 149:2509-11.

7 Poller L. United Kingdom External Quality Assessment in blood coagulation: the first 21 years. J Clin Pathol 1989;42:1-3.

8 Bland JM, Altman DG. Statistical methods for assessing agreement between two methods of clinical measurement. Lancet 1986;1:307-10.

9 Taberner DA, Poller L, Thompson JM, Darby KV. Effect of international sensitivity index (ISI) of thromboplastins on precision of international normalised ratios. J Clin Pathol 1989;42:92-6. 